\title{
LETTERS
}

\section{After hours surgery and mortality: the potential role of acute care surgery models as a factor accounting for results}

We read with interest the article by Sheehan and colleagues ${ }^{1}$ regarding hip fracture mortality varying by setting. We wondered whether it were possible to determine from their data if time of day of surgery, which is an important risk factor in morbidity and mortality, was a factor.

Wang and colleagues ${ }^{2}$ showed that the odd ratios (ORs) of mortality for afterhours surgery (compared with those for regular hours) are significantly increased. They report the OR for mortality (when compared with surgery during regular hours) as 1.43 for surgery starting from 15:30-23:29 and as 2.17 when starting from 23:30-07:29.

Academic and large community hospitals are more likely than smaller centres to have daytime orthopedic trauma rooms, emergency operating rooms and available, dedicated surgical personnel. In centres without daytime emergency operating rooms, the general trend is for emergency cases that can wait (such as hip fracture) to do so until after elective procedures are completed and only then to proceed, which results in potential delays in care, and operations occurring at a riskier time. ${ }^{3}$

Systems like the acute care surgery service $^{4,5}$ and the dedicated orthopedic trauma room ${ }^{3}$ have been shown to significantly reduce after-hours emergency surgeries. One system was shown to reduce afterhours surgeries (defined as occurring from 4:30 pm-7:00 am) for femoral neck fractures from $66.7 \%$ to $19.3 \% .^{6}$ This alone could decrease mortality for frail, elderly patients.

This raises a potential solution, not considered in the article, ${ }^{1}$ for smaller centres: the provision of and funding for daytime dedicated emergency operating rooms and the surgical staff to provide service or transfers (to centres with these systems) for patients with hip fractures.

\section{David R. Lardner MBChB}

Pediatric anesthesiologist, Alberta

Children's Hospital, University of Calgary, Calgary, Alta.

\section{Carmen A. Brauer MD MSc}

Orthopedic surgeon, University of

Calgary, Calgary, Alta.

\section{A. Rob Harrop MD MSc}

Plastic surgeon, Alberta Children's Hospital, University of Calgary, Calgary, Alta.

\section{Ali MacRobie}

Research assistant, EQuIS, Alberta Children's Hospital, University of Calgary, Calgary, Alta.

All authors are members of EQuIS, Alberta Children's Hospital and the University of

Calgary, Calgary, Alta.

Cite as: CMAJ 2017 February 6;189:E219. doi: 10.1503/cmaj.732681

\section{References}

1. Sheehan KJ, Sobolev B, Guy P, et al. In-hospital mortality after hip fracture by treatment setting. CMAJ 2016;188:1219-25.

2. Wang NN, Tessler MJ, Charland L. Retrospective analysis of time of day of surgery and its 30 day in-hospital postoperative mortality rate at a single Canadian institution [abstract]. Anesth Analg 2016;123(Suppl):764-5.

3. Min W, Wolinsky PR. The dedicated orthopedic trauma operating room. J Trauma 2011;71: 513-5.

4. Wanis KN, Hunter AM, Harington MB, et al. Impact of an acute care surgery service on timeliness of care and surgeon satisfaction at a Canadian academic hospital: a retrospective study. World J Emerg Surg 2014;9:4.

5. Parasyn AD, Truskett PG, Bennett M, et al. Acutecare surgical service: a change in culture. ANZ J Surg 2009;79:12-8.

6. Roberts TT, Vanushkina M, Khasnavis S, et al. Dedicated orthopaedic operating rooms: beneficial to patients and providers alike. J Orthop Trauma 2015;29:e18-23.

Competing interests: None declared. 